\title{
Kinematic evaluation of the whiffletree lateral support setup and its application for the mirror support of the TMT tertiary mirror
}

\author{
W. Fu-guo \\ wfg109@163.com
}

\section{Z. Linmin}

X. Jin

\begin{abstract}
Changchun Institute of Optics, Fine Mechanics and Physics ,Chinese Academy of Sciences, Photoelectric detection department, Dong Nanhu Road 3888, Changchun Jilin, 130033, PRC

Changchun Institute of Optics, Fine Mechanics and Physics ,Chinese Academy of Sciences, Photoelectric detection department, Dong Nanhu Road 3888, Changchun Jilin, 130033, PRC

College of Mechanical and Electronic Engineering ,Shandong University of Science and Technology, Qingdao, 266590, PRC

Department of Electromechanical Engineering, Binzhou University, Binzhou 256600, Shandong Province, PRC
\end{abstract}

With increasing size of the reflecting mirror apertures in telescopes, the lateral support is becoming more and more important. In order to find a setup satisfying the requirements for the TMT tertiary mirror, based on the classic three-point flexure support setup, a twelve-point lateral support setup was investigated its basic internal relations and properties were investigated. The virtual equivalent circle concept is then proposed to evaluate different potential setups. By studying the relationship between the virtual equivalent circle radius and the thermal deformation and resonant frequency, it can be concluded that a larger virtual equivalent circle radius results in a smaller the thermal deformation and a higher resonant frequency. Preliminary research results suggested that the TMT tertiary mirror surface figure error and thermal deformation requirements can easily be met. However, it was more difficult to obtain resonant frequencies higher than $15 \mathrm{~Hz}$. Based on the virtual equivalent circle concept, the TMT tertiary mirror support setup was optimized, resulting in a resonant frequency of $17.7 \mathrm{~Hz}$, which satisfies the requirements. The whiffletree lateral support setup can be applied to similar reflecting mirror support structures. [DOI: http://dx.doi.org/10.2971/jeos.2015.15027]

Keywords: Kinematic, whiffletree, lateral support, TMT tertiary mirror

\section{INTRODUCTION}

With the current developments in astronomy, in order to observe objects more clearly and objects farther away, scientists and engineers aim to manufacture even larger telescopes. Large aperture telescopes currently in production are the TMT (Thirty Meter Telescope), the GMT (Giant Magellan Telescope) and the EELT (European Extremely Large Telescope). China participates in the manufacturing of the TMT, and CIOMP is responsible for the TMT tertiary mirror system.

The TMT tertiary mirror is an elliptical flat mirror; its major axis is $3594 \mathrm{~mm}$ long, the minor axis is $2586 \mathrm{~mm}$ long and its thickness is $100 \mathrm{~mm}$. The axial support is based on an 18 point whiffletree structure. For the lateral support, there are several options available $[1,2]$, i.e. a mercury bag support, a counterweighted lever support, and a hydraulic or pneumatic pressure support.

The TMT tertiary mirror is located in the optical beam path. Therefore, the application of a mercury bag support and a hydraulic or pneumatic pressure support structure is complicated, and the counterweighted lever support frequency is low. Thus, these types of support structure cannot meet the necessary requirements, and a new type of lateral support structure is needed, which meets the requirements concern- ing structure design allocation, structure design weight and resonant frequency.

\section{PRINCIPLES OF THE WHIFFLETREE LATERAL SUPPORT STRUCTURE}

\subsection{Principles of the classic kinematic lateral support structure}

A detailed introduction into the physics of small aperture lenses and reflective mirror support structures was given by Yoder [1]. Figure 1 shows the classic kinematic three-point support concept, which is the basic and classic kinematic support structure. Three flexure bearings are laterally connected to the mirror mount. The curve arrowheads illustrate the movement direction if only one flexure support is working. In the ideal case, the free movement lines should intersect at one point, the flexure lengths are equal, and the three flexure bearings form an equilateral triangle.

The function of this flexure support system can be explained as follows: Without the flexure support $C$, the flexure support $\mathrm{A}$ and $\mathrm{B}$ allow the mirror to rotate around point $\mathrm{O}$, where $\mathrm{O}$ is the intersect point of the flexure support $\mathrm{A}$ and $\mathrm{B}$. Point $\mathrm{O}$ and point $C$ are coplanar. Since the flexure bearing $C$ is rigid in this direction, it becomes impossible for the mirror to rotate around point $\mathrm{O}$. 


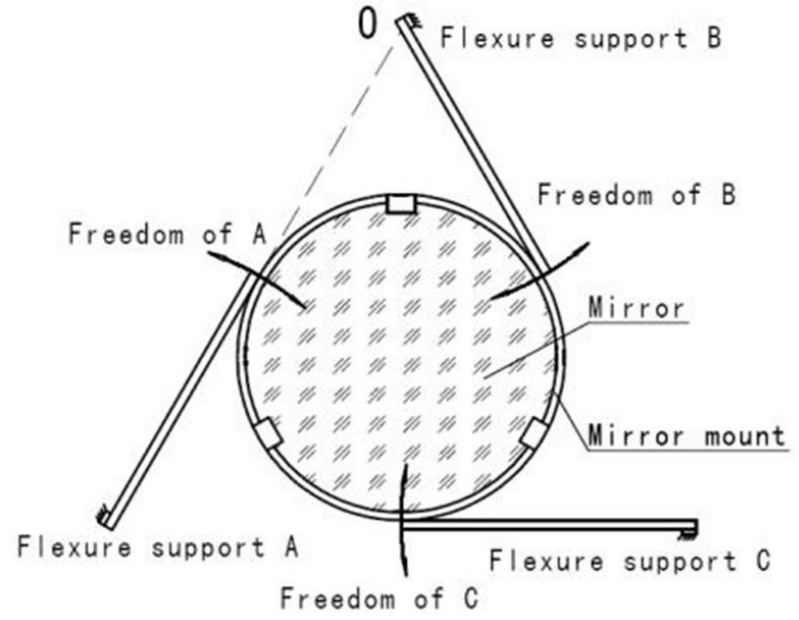

FIG. 1 Schematic illustration of a classic kinematic three-point mirror support structure.

The three flexure bearings constitute an equilateral triangle, resulting in a high resonant frequency, and it is important that the thermal deformation is small. Even if the alternate temperature causes expansion or shrinkage, it can only produce radial motion and no internal stress. The unique mirror motion is a rotation around the surface normal. This kind of flexure support is widely used for small aperture mirrors.

\subsection{Kinematic multi-point lateral support}

When the reflective mirror aperture becomes larger and larger, the surface figure error requirements can no longer be satisfied by using three lateral support points, and more lateral support points are needed. Based on the three lateral point principles discussed in Section 2.1, each support point can be divided into two points, three points or four points etc. Thus, the basic three-point lateral support can be changed into a six-point, nine-point or twelve-point support [3,4].

For the TMT tertiary mirror, professor Eirc Williams suggested to adopt a twelve-point lateral support structure. Therefore, the twelve-point lateral support was adopted for this study, and its internal relations and properties are discussed in detail. The support structure must adhere to the following conditions:

\section{All lateral support rods are flexure.}

2. The lateral support points are divided into three groups, the support forces in each group are parallel, and their value is equal.

3. The three groups of support forces enclose angles of $120^{\circ}$.

4. There is a connecting link in the center of the support arms. Each connecting link can be rotated, such as the round spot shown in Figure 2.

According to these four points, the four forces in each group satisfy the balanced lever principle. According to the force equivalence and simplification principles, the four forces in each group can be simplified to one force such as the imaginary line in Figure 2. This equivalence force is four times

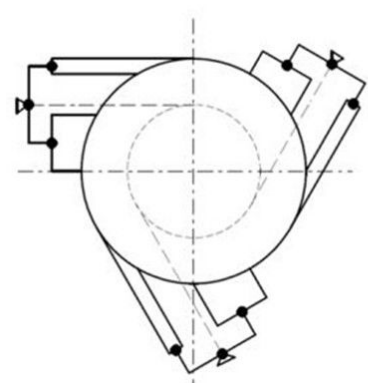

(a)

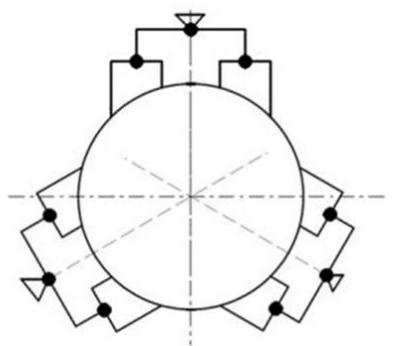

(b)
FIG. 2 Schematic illustration of two possible topologies for a twelve-point lateral support structure.

as large as each support force. The triangle composed of the three equivalence forces is similar to the classic kinematic lateral support. Therefore, the twelve-point lateral support can be treated as a modification of the classic lateral support structure.

\section{VIRTUAL EQUIVALENCE CIRCLE}

There are different possible topologies for realizing a twelvepoint lateral support. As an example, Figure 2(b) shows another topology type. To compare the different topology types, the virtual equivalence circle concept is applied. First, all support forces are simplified into three equivalent forces, with angles of $120^{\circ}$ between the three equivalent forces, resulting in an equilateral triangle. The circle which can be inscribed into the equilateral triangle is defined as the virtual equivalence circle. We can compare the advantages and disadvantages of the different topologies by studying the virtual equivalence circle.

\subsection{Relationship between the virtual equivalence circle and the thermal deformation}

The virtual equivalence circle radius is approx. $0.6 \mathrm{R}$ for the setup in Figure 2(a) ( $R$ is the mirror radius), and the support forces are tangential to the virtual equivalence circle. When the temperature changes, thermal expansion and shrinking of the support rods only cause the reflective mirror to rotate around the center of the virtual equivalence circle. This rotation does not affect the mirror surface figure error.

Concerning the thermal deformation, the adaptability for thermal deformation of the setup in Figure 2(a) is strong. In contrast, the virtual equivalence circle radius is close to zero for the setup in Figure 2(b). In other words, the equivalence forces intersect in the center of the reflective mirror. When the temperature changes, due to the different thermal expansion coefficients of the support rod and the mirror, the reflective mirror will stretch and extrude, resulting in stress in the mirror and an increase of the mirror surface figure error. Therefore, the adaptability for thermal deformation of the setup shown in Figure 2(b) is weak. 


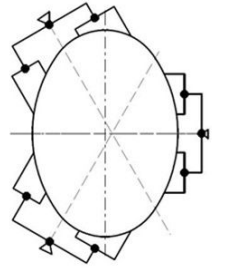

(a)

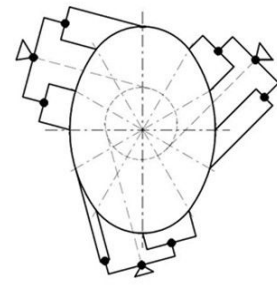

(b)

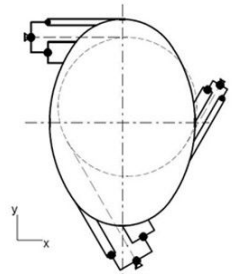

(c)
FIG. 3 Comparison of three potential setups for the TMT tertiary mirror lateral support structure.

\subsection{Relationship between the virtual equivalence circle and the resonant frequency}

An evaluation of the support performance must not only consider the surface figure error, but also the resonant frequency. The higher the resonant frequency, the easier it is to stabilize and control the mirror. For the type of structure shown in Figure 1 , the radius of the virtual equivalence circle is considered to be $R$, i.e. equivalent to the mirror radius). For the type of structure shown in Figure 2(a), the radius of the virtual equivalence circle is considered as $0.6 \mathrm{R}$, and for the type of structure shown in Figure 2(b), the radius of the virtual equivalence circle is considered to be zero.

If the radius of the virtual equivalence circle is zero, one might think that the mirror is unstable, and thus the resonant frequency approaches zero. For the type of structure shown in Figure 1, the resonant frequency is highest, and the resonant frequency obtained for the type of structure shown in Figure 2 is between the frequencies obtained for the structures shown in Figure 1 and Figure 2(b). Concerning the design of the mirror support structure, we expect that the structure benefits from a large virtual equivalence circle radius. Thus, the objective for optimizing the support structure topology is to maximize the virtual equivalence circle radius.

\section{APPLICATION OF THE WHIFFLETREE LATERAL SUPPORT STRUCTURE}

The TMT tertiary mirror is an elliptical flat mirror, and a mirror resonant frequency higher than $15 \mathrm{~Hz}$ is required $[5,6]$. Lateral support is provided by a twelve-point whiffletree support structure.

In this section, three different possible topologies are compared. The lateral support points are uniformly distributed for the setups shown in Figures 3(a) and 3(b). In contrast, the lateral support points are not uniformly distributed for the setup shown in Figure 3(c). The aim is to find the maximum virtual equivalence circle radius.

According to the three potential plans, the TMT tertiary mirror FE model is built, three different lateral support structures are analysed [7]. Table 1 is the FEA result.

Note: The resonant frequency is related to the diameter of the

\begin{tabular}{|l|c|c|c|}
\hline & $a$ & $b$ & $c$ \\
\hline \hline Surface figure & $P V=$ & $P V=$ & $P V=$ \\
error(gravitational & $205 \mathrm{~nm}$ & $190.8 \mathrm{~nm}$ & $145.6 \mathrm{~nm}$ \\
force parallel to the & $R M S=$ & $R M S=$ & $R M S=$ \\
mirror major axis) & $34.4 \mathrm{~nm}$ & $22.7 \mathrm{~nm}$ & $15.0 \mathrm{~nm}$ \\
\hline Surface figure & $P V=$ & $P V=$ & $P V=$ \\
error when & $45 \mathrm{~nm}$ & $17 \mathrm{~nm}$ & $8 \mathrm{~nm}$ \\
temperature is & $R M S=$ & $R M S=$ & $R M S=$ \\
changed by 10 K & $9.2 \mathrm{~nm}$ & $2.9 \mathrm{~nm}$ & $1.6 \mathrm{~nm}$ \\
\hline Resonant & & & \\
frequency & $0.7 \mathrm{~Hz}$ & $9.8 \mathrm{~Hz}$ & $17.7 \mathrm{~Hz}$ \\
\hline
\end{tabular}

TABLE 1 Comparison of the FEA results for the three different TMT tertiary mirror lateral support structure setups.

lateral support rods. In the FE model, the diameter of all lateral support rods is $3 \mathrm{~mm}$.

As shown in Table 1, the virtual equivalence circle radius affects the surface figure error, the thermal deformation and the resonant frequency $[8,9]$. The setup shown in Figure 3(a) is not only greatly affected by a change in temperature but also the resonant frequency is close to zero. The resonant frequency obtained for the setup shown in Figure 3(b) satisfies the requirements, and the setup shown in Figure 3(c) can strictly meet the requirements.

In fact, the virtual equivalence circle radius can still be increased for the setup in Figure 3(c), and thus its resonant frequency can be higher as well. However, with increasing virtual equivalence circle radius, the distance between the support points in one group is reduced, which makes the design allocation small, and results in a higher local stress. Therefore, the setup proposed in Figure 3(c) represents the best possible mirror support structure compared to the other two proposed support structures [10]-[12]. Figure 4 is the TMT tertiary mirror support conceptual design [12] according to the principle of Figure 3(c), and Figure 5, Figure 6 and Figure 7 are the Figure 4 corresponded partial enlarged picture.

\section{SUMMARY}

In order to find a setup satisfying the requirements for the lateral support of the TMT tertiary mirror, based on the classic kinematic three-point lateral support model, the twelve-point whiffletree lateral support setup and its basic characteristics were investigated. The virtual equivalence circle concept was proposed, and the relationship between the virtual equivalence circle and the thermal deformation as well as the resonant frequency has been studied.

In conclusion, the mirror's thermal deformation decreases and its resonant frequency increases with increasing radius of the virtual equivalence circle. The virtual equivalence circle concept was applied to evaluate different concepts for the mirror support of the TMT tertiary mirror. The best setup resulted in a resonant frequency of $17.7 \mathrm{~Hz}$, which satisfies the requirements. This lateral support setup can also be applied to the support structure of similar mirrors. 


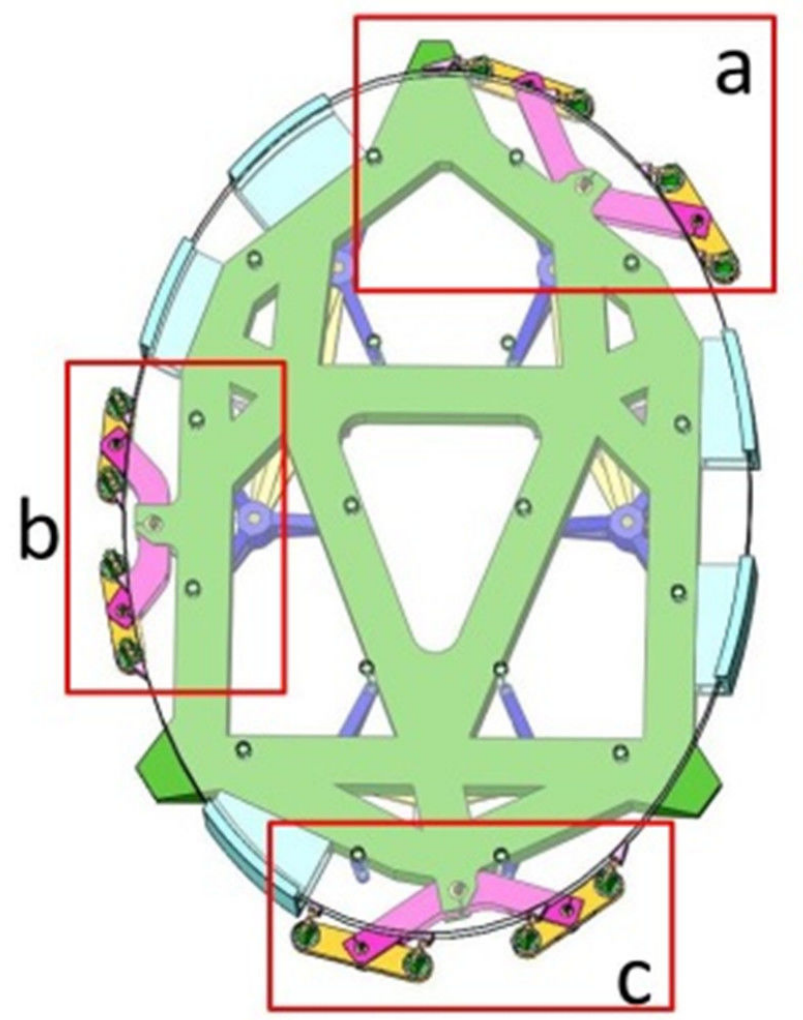

FIG. 4 Schematic illustration showing the conceptual design of the TMT tertiary mirror support structure.

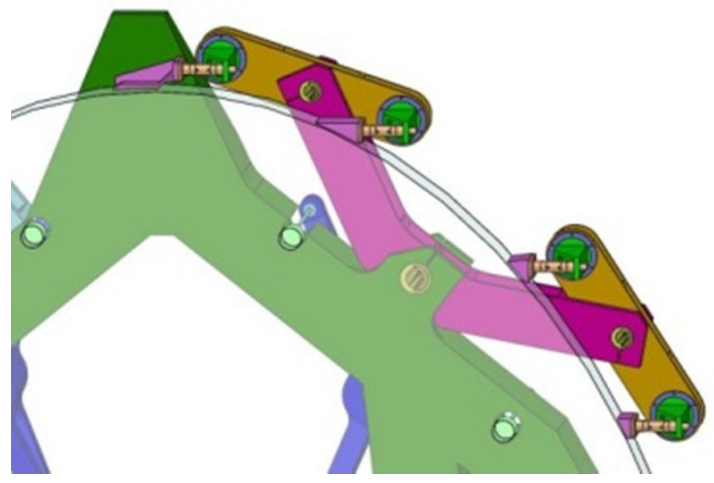

FIG. 5 Partial enlarged picture of label a in Figure 4.

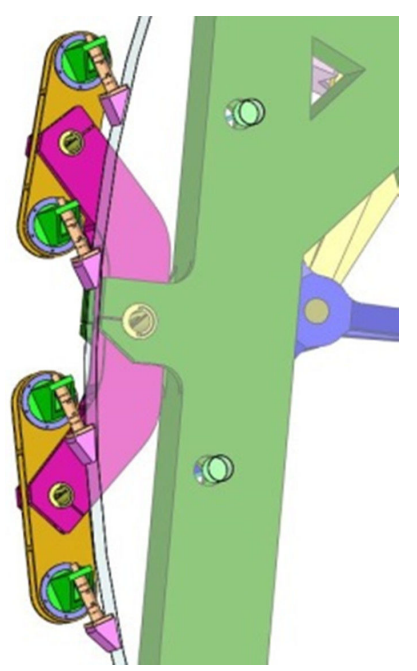

FIG. 6 Partial enlarged picture of label b in Figure 4.

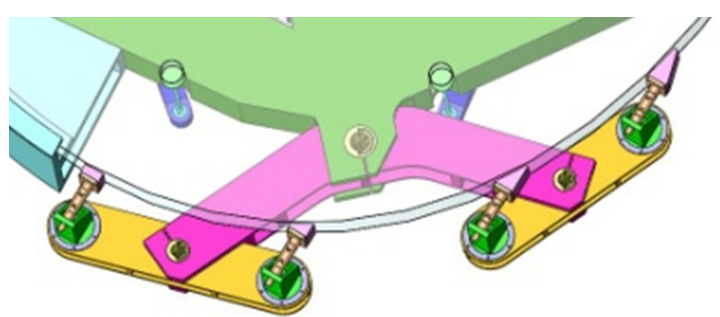

FIG. 7 Partial enlarged picture of label $c$ in Figure 4.

\section{ACKNOWLEDGEMENT}

This work was supported by National Natural Science Foundation of China (Nos. 11403023). The author wants to thank TMT expert Eric Williams for his continuous support, and all staff at CTMT for their cooperation.

\section{References}

[1] P. R. Yoder Jr., Opto-mechanical systems design (Taylor a Francis Group, Boca Raton, 2006)

[2] L. Zongxuan, J. Guang, Zh. Lei, and K. Lin, "Overview and outlook of monolithic primary mirror of spaceborne telescope with $3.5 \mathrm{~m}$ aperture (in Chinese)," Optics and Precision Engineering 7(4), 532-541 (2014).

[3] F. Jia, Zh. Jingxu, W. Fuguo, Y. Fei, and X. Wei, "Application of Whiffletree for Primary Mirror Lateral Support (in Chinese)," Infrared Technology 34(4), 209-216 (2012).

[4] Y. Xinfeng, G. Yan, N. Mingyang, and Q. Suo, "Optical surface figure of lens under kinematic mount apparatus and its reproducibility (in Chinese)," Optics and Precision Engineering 21(8), 2000-2007 (2013).

[5] TMT Group, "Tertiary mirror surface figure specification," TMT.OPT.SPE.12.001.DRF02 (2012).

[6] TMT Group, "Design requirements document for tertiary mirror system (M3S)," TMT.OPT.DRD.07.006.REL29 (2012).

[7] M. K. Cho, "Performance prediction of the TMT tertiary mirror support system," Proc. SPIE 7018, 70184F-1 (2008).

[8] W. Fu-guo, Y. Fei, and Zh. Jing-xu, "TMT tertiary mirror passive support system conceptual design," Infrared and Laser Engineering 5 , 1269-1274 (2013).

[9] W. Fuguo, Y. Fei, Zh. Hongchao, S. Yanqin, and Ch. Baogang, "Progress in TMT M3 system (in Chinese)," Chinese Optics 6(5), 643-651 (2013).

[10] S. Xiaogang, S. Ning, Zh. Renshan, and Q. Yanfeng, "Design of supporting structure for primary mirror of larger aperture theodolite (in Chinese)," Optics and Precision Engineering 21(12), 3111-3117 (2013).

[11] X. Hong, and G. Yingjun, "Structure design of $1 \mathrm{~m}$ diameter space mirror component of space camera (in Chinese)," Optics and Precision Engineering 21(6), 1488-1495 (2013).

[12] X. Xinhang, G. Yunguo, Y. Hongbo, W. Bing, and H. Xudong, "Largediameter fast steering mirror on rigid support technology for dynamic platform (in Chinese)," Optics and Precision Engineering 22(1), 117-124 (2014). 